\title{
Reproducing Kernels and Radial Differential Operators for Holomorphic and Harmonic Besov Spaces on Unit Balls: a Unified View
}

\author{
H. Turgay Kaptanoğlu \\ (Communicated by Alexander Yu. Solynin)
}

\begin{abstract}
We investigate some relations between the reproducing kernels of Hilbert spaces of holomorphic and harmonic functions on the unit balls and the radial differential operators acting on the spaces that allow their characterization via integrals of their derivatives on the balls. We compare and contrast the holomorphic and harmonic cases.
\end{abstract}

Keywords. Besov, Dirichlet, Drury-Arveson, Hardy, Bergman space, reproducing kernel Hilbert space, radial differential operator, spherical harmonic.

2000 MSC. 46E22, 46E20, 46E15, 26A33, 32A37, 32A36, 31B05, 31C25, $33 \mathrm{C} 55,47 \mathrm{~B} 34$.

\section{Introduction}

The purpose of this paper is to make a short survey of the most basic characteristics of the two-parameter Besov spaces of holomorphic and harmonic functions on the unit balls with standard weights by stressing the similarities between the two categories and by paying particular attention to the reproducing kernels of those that are Hilbert spaces and to the radial differential operators that are so convenient in defining the spaces via integral norms.

We restrict our attention strictly to spaces with Bergman-type norms, and do not mention any spaces with Bloch-type norms. We also omit from consideration the full three-parameter Besov family. So we do not mention any Hardy-Sobolev spaces or spaces with Hardy-Bergman mixed-type norms. The exposition is aligned with the interests and the research of the author. No attempt is made to give exact references for classical results.

Received February 9, 2010.

Published online July 28, 2010.

This research is partially supported by TÜBITAK under Research Project Grant 108T329. 


\section{Notation and preliminaries}

We start by introducing the notation used throughout the paper. The Pochhammer symbol $(a)_{b}$ for $a, b \in \mathbb{C}$ is defined by

$$
(a)_{b}=\frac{\Gamma(a+b)}{\Gamma(a)}
$$

when $a$ and $a+b$ are off the pole set $-\mathbb{N}$ of the gamma function $\Gamma$. This is a shifted factorial since $(a)_{k}=a(a+1) \cdots(a+k-1)$ for positive integer $k$. In particular, $(1)_{k}=k !$ and $(\cdot)_{0}=1$. The Pochhammer symbol seems more useful than the gamma function itself because of its more controlled polynomial growth as one of its parameters increases without bound; see [24]. The Stirling formula gives

$$
(c)_{b} \sim c^{b}, \quad \frac{(c)_{a}}{(c)_{b}} \sim c^{a-b} \text { and } \quad \frac{(a)_{c}}{(b)_{c}} \sim c^{a-b} \quad \text { as } \operatorname{Re} c \rightarrow \infty
$$

where $A \sim B$ means that $|A / B|$ is bounded above and below by two positive constants. Such constants that are independent of the parameters in the equation are all denoted by the generic unadorned upper case $C$.

We alternately work in $\mathbb{C}^{N}$ and $\mathbb{R}^{n}$, where $N \geq 1$ and $n \geq 2$. Equating $n=2 N$ allows for a comparison in even dimensions. The Hermitian inner product and the norm of $\mathbb{C}^{N}$ are $\langle z, w\rangle=z_{1} \bar{w}_{1}+\cdots+z_{N} \bar{w}_{N}$ and $|z|=\sqrt{\langle z, z\rangle}$. The inner (dot) product and the norm of $\mathbb{R}^{n}$ are $x \cdot y=x_{1} y_{1}+\cdots+x_{n} y_{n}$ and $|x|=\sqrt{x \cdot x}$. We denote the unit balls and the unit spheres with respect to $|\cdot|$ in $\mathbb{C}^{N}$ or $\mathbb{R}^{n}$ by $\mathbb{B}$ and $\mathbb{S}$. We often write $z=R \zeta, w=r \omega \in \mathbb{C}^{N}, x=R \xi, y=r \eta \in \mathbb{R}^{n}$ with $R=|z|$ or $R=|x|, r=|w|$ or $r=|y|$, and $\zeta, \omega, \xi, \eta \in \mathbb{S}$. When $N=1$ or $n=2$, we talk about the unit disc $\mathbb{D}$ and the unit circle $\mathbb{T}$ instead.

We denote by $H(\mathbb{B})$ the space of holomorphic functions $f$ on $\mathbb{B} \in \mathbb{C}^{N}$, and by $h(\mathbb{B})$ the space of harmonic functions $u$ on $\mathbb{B} \in \mathbb{R}^{n}$, both with the Fréchet topology of uniform convergence on compact subsets. Members of $h(\mathbb{B})$ are the complex-valued real-analytic functions annihilated on $\mathbb{B}$ by the usual Laplacian.

Let $\nu$ be the volume measure on $\mathbb{B}$ normalized as $\nu(\mathbb{B})=1$, and let $\sigma$ be the surface measure on $\mathbb{S}$ normalized as $\sigma(\mathbb{S})=1$. For $q \in \mathbb{R}$, define on $\mathbb{B}$ also the measures

$$
d \nu_{q}(\cdot)=C_{q}\left(1-|\cdot|^{2}\right)^{q} d \nu(\cdot) .
$$

These measures are finite only for $q>-1$ and then we choose $C_{q}$ so that $\nu_{q}(\mathbb{B})=1$. For $q \leq-1$, we set $C_{q}=1$. We denote the Lebesgue classes with respect to $\nu_{q}$ by $L_{q}^{p}$. The exact value of $C_{q}$ is

$$
C_{q}=\frac{(1+q)_{n / 2}}{(1)_{n / 2}}=\frac{(1+n / 2)_{q}}{(1)_{q}}, \quad q>-1 .
$$

Thus $C_{q} \sim n^{q}$ for $n$ large, and $C_{q} \sim q^{n / 2}$ for $q$ large. To use this in $\mathbb{C}^{N}$, we just replace $n / 2$ by $N$. Also $C_{q}=1+q$ when $n=2(N=1)$ and naturally $C_{0}=1$. 
We use multi-index notation in which $\alpha=\left(\alpha_{1}, \ldots, \alpha_{N}\right) \in \mathbb{N}^{N}$ is an $N$-tuple of non-negative integers, $|\alpha|=\alpha_{1}+\cdots+\alpha_{N}, \alpha !=\alpha_{1} ! \cdots \alpha_{N}$ !, $z^{\alpha}=z_{1}{ }^{\alpha_{1}} \cdots z_{N}{ }^{\alpha_{N}}$ for $z=\left(z_{1}, \ldots, z_{N}\right) \in \mathbb{C}^{N}$, and $0^{0}=1$. To use them in $\mathbb{R}^{n}$, we just change $N$ to $n$ and $z$ to $x$.

Proposition 2.1. We have the orthogonality relation

$$
\int_{\mathbb{S}} \zeta^{\alpha} \bar{\zeta}^{\beta} d \sigma(\zeta)=0, \quad \alpha \neq \beta
$$

and the exact value

$$
\int_{\mathbb{S}}\left|\zeta^{\alpha}\right|^{2} d \sigma(\zeta)=\frac{\alpha !}{(N)_{|\alpha|}}
$$

A proof is given in [23, Prop. 1.4.8 and 1.4.9].

A complex-valued polynomial $p$ of the real or complex variable $v=\left(v_{1}, v_{2}, \ldots\right)$ is called homogeneous of degree $l$ if $p(\tau v)=\tau^{l} p(v)$ for $\tau \in \mathbb{R}$. Its gradient is $\nabla p=\left(\partial p / \partial v_{1}, \partial p / \partial v_{2}, \ldots\right)$, and its usual radial derivative is

$$
\mathcal{R} p(v)=v \cdot \nabla p(v)=\frac{d}{d \tau}[p(\tau v)]_{\tau=1} .
$$

By homogeneity,

$$
\mathcal{R} p=l p .
$$

Using the Pochhammer symbol, the binomial expansion is written as

$$
\frac{1}{(1-z)^{Q}}=\sum_{k=0}^{\infty} \frac{(Q)_{k}}{(1)_{k}} z^{k}, \quad Q>0, z \in \mathbb{D} .
$$

It is a special case of the classical hypergeometric function

$$
{ }_{2} F_{1}(a, b ; c ; z)=\sum_{k=0}^{\infty} \frac{(a)_{k}(b)_{k}}{(c)_{k}(1)_{k}} z^{k}, \quad a, b \in \mathbb{R}, c>0, z \in \mathbb{D},
$$

which we use with suitable values of $a, b, c$ depending on $Q$ to extend the region of validity of the binomial expansion to $Q \leq 0$. Both expansions converge absolutely and uniformly on compact subsets of $\mathbb{D}$, and thus both functions belong to $H(\mathbb{D})$. We also have their variants belonging to $h(\mathbb{D})$ below.

We denote an integral inner product on a space $X$ of functions on a set $E$ by $[\cdot, \cdot]_{X}$ and the corresponding norm on it by $\|\cdot\|_{X}$.

Definition 2.2. We call a function $K(\cdot, \cdot)$ on $E \times E$ a reproducing kernel for a Hilbert space $X$ of functions defined on $E$ if $K(\lambda, \cdot) \in X$ for each $\lambda \in E$ and

$$
g(\lambda)=[g(\cdot), K(\lambda, \cdot)]_{X}, \quad g \in X, \lambda \in E .
$$

For more on reproducing kernel Hilbert spaces including the following results, see $[1$, Ch. 2]. The first one is just the Riesz lemma together with its converse. 
Theorem 2.3. A Hilbert space $X$ of functions on $E$ admits a reproducing kernel if and only if the point evaluation functional $g \mapsto g(\lambda)$ at every $\lambda \in E$ is a non-zero continuous functional on $X$.

Theorem 2.4. A sesquilinear function $K(\cdot, \cdot)$ is a reproducing kernel on $E$ if and only if it is positive semidefinite there and non-zero on the diagonal of $E \times E$. Such a function generates a unique Hilbert space of functions on $E$ whose members are of the same class as $K(\lambda, \cdot)$.

That $K$ is positive semidefinite means that

$$
\sum_{j=1}^{l} \sum_{k=1}^{l} \bar{a}_{j} a_{k} K\left(\lambda_{j}, \lambda_{k}\right) \geq 0
$$

for $a_{1}, \ldots, a_{l} \in \mathbb{C}$ and distinct $\lambda_{1}, \ldots, \lambda_{l} \in E$. Sesquilinearity reduces to bilinearity for real-valued functions.

Corollary 2.5. An infinite sum of reproducing kernels on $\mathbb{B}$ for subspaces of $H(\mathbb{B})$ or $h(\mathbb{B})$ converging uniformly on compact subsets of $\mathbb{B} \times \mathbb{B}$ is also a reproducing kernel on $\mathbb{B}$ for a Hilbert subspace of $H(\mathbb{B})$ or $h(\mathbb{B})$.

Theorem 2.6. Suppose $X$ is a Hilbert space with reproducing kernel $K$ and that $\left\{e_{1}, e_{2}, \ldots\right\}$ is an orthonormal basis for $X$. Then $K(\lambda, \mu)=\sum_{j=1}^{\infty} e_{j}(\lambda) \bar{e}_{j}(\mu)$.

\section{Spherical harmonics}

For any $N \geq 1$, members of $H(\mathbb{B})$ are described using power series in one or more variables. The same is true for members of $h(\mathbb{D})$, where the extensions of the Fourier series on $\mathbb{T}$ to the disc suffice. However, for members of $h(\mathbb{B})$ with $n>2$, there is something more elaborate which extends what is known for $n=2$.

For $m=0,1,2, \ldots$, let $\mathcal{H}_{m}$ be the space of complex-valued polynomials of the form $\sum_{|\alpha|=m} b_{\alpha} x^{\alpha}$ on $\mathbb{R}^{n}$ that are also harmonic; such polynomials are homogeneous of degree $m$. Functions in $\mathcal{H}_{m}$ are determined by their restrictions to $\mathbb{S}$ by homogeneity; these restrictions are called spherical harmonics; we identify restricted and non-restricted spaces. Clearly, each $\mathcal{H}_{m}$ is finite dimensional with dimension

$$
h_{m}=\operatorname{dim} \mathcal{H}_{m}=\frac{n-2+2 m}{m} \frac{(n-1)_{m-1}}{(1)_{m-1}} \sim m^{n-2}, \quad m \rightarrow \infty .
$$

Hence each $\mathcal{H}_{m}$ is a closed subspace of $L^{2}(\sigma)$ and thus a Hilbert space with respect to the inner product of $L^{2}(\sigma)$.

Similar to the theory of Fourier series, we have

$$
L^{2}(\sigma)=\bigoplus_{m=0}^{\infty} \mathcal{H}_{m}
$$


which is an orthogonal direct sum, meaning that $\mathcal{H}_{m}$ is orthogonal to $\mathcal{H}_{k}$ for $m \neq k$ with respect to the inner product of $L^{2}(\sigma)$, and every function in $L^{2}(\sigma)$ can be expanded in a unique series of polynomials in the $\mathcal{H}_{m}$ converging in $L^{2}(\sigma)$.

The point evaluation functional at each point $\eta \in \mathbb{S}$ trivially is a bounded linear functional on $\mathcal{H}_{m}$. Thus, by Theorem 2.3 , there is a unique function $Z_{m}(\cdot, \eta) \in \mathcal{H}_{m}$ such that

$$
u(\eta)=\int_{\mathbb{S}} f(\cdot) Z_{m}(\cdot, \eta) d \sigma, \quad u \in \mathcal{H}_{m} .
$$

In other words, $Z_{m}$ is the reproducing kernel of $\mathcal{H}_{m}$; it is called the zonal harmonic of degree $m$. It is real-valued and symmetric in its two variables. When $m=0$, $\mathcal{H}_{0}$ is just the constants, $h_{0}=1$, and $Z_{0} \equiv 1$.

Other properties of zonal harmonics are that

$$
\left|Z_{m}(\xi, \eta)\right| \leq Z_{m}(\xi, \xi)=h_{m}, \quad \xi, \eta \in \mathbb{S} ;
$$

that $Z_{m}(T(\xi), T(\eta))=Z_{m}(\xi, \eta)$ for any orthogonal transformation $T$; and that $Z_{m}(\xi, \cdot)$ is constant on each parallel of $\mathbb{S}$ orthogonal to $\xi$; all for any $\xi, \eta \in \mathbb{S}$.

Let $\left\{Y_{m 1}, \ldots, Y_{m h_{m}}\right\}$ be a basis for $\mathcal{H}_{m}$ that is orthonormal with respect to the inner product of $L^{2}(\sigma)$. Then by Theorem 2.6,

$$
Z_{m}(\xi, \eta)=\sum_{j=1}^{h_{m}} Y_{m j}(\xi) \bar{Y}_{m j}(\eta)
$$

The spaces $\mathcal{H}_{m}$, the functions in them, the $Z_{m}$, and the $Y_{m j}$ are extended to $\mathbb{B}$ by homogeneity; for example, $Z_{m}(x, y)=R^{m} r^{m} Z_{m}(\xi, \eta)$. Such extensions are sometimes called solid spherical harmonics. It is well-known that the Poisson kernel $\mathcal{P}$ for $\mathbb{B}$ is the sum of all zonal harmonics. This idea also extends $\mathcal{P}$ to $\mathbb{B}$ in both variables. Thus we have

$$
\begin{aligned}
\mathcal{P}(x, y) & =\sum_{m=0}^{\infty} Z_{m}(x, y)=\sum_{m=0}^{\infty} \sum_{j=1}^{h_{m}} Y_{m j}(x) \bar{Y}_{m j}(y) \\
& =\frac{1-R^{2} r^{2}}{\left(1-2 x \cdot y+R^{2} r^{2}\right)^{n / 2}}, \quad x, y \in \mathbb{B} .
\end{aligned}
$$

The picture equivalent to spherical and zonal harmonics for $H(\mathbb{B})$ is much simpler and more explicit. Spherical harmonics correspond to the space of all holomorphic polynomials of the form

$$
\sum_{|\alpha|=k} b_{\alpha} z^{\alpha}
$$

whose dimension is

$$
\theta_{k}=\frac{(N)_{k}}{(1)_{k}} \sim k^{N-1}, \quad k \rightarrow \infty
$$


Further, an integration similar to (8) with $f(\zeta)=\zeta^{\alpha}$ using Proposition 2.1 and the uniqueness of kernels show that the counterparts of zonal harmonics are the sesquilinear functions

$$
W_{k}(\zeta, \omega)=\frac{(N)_{k}}{(1)_{k}}\langle\zeta, \omega\rangle^{k}
$$

This also implies that the $W_{k}$ are positive kernels on $\mathbb{S}$. The equivalent of $(7)$ is obvious by the same proposition.

Other properties of zonal harmonics carry over here too, and in fact trivially due to the explicit form of the $W_{k}$. We have that $\left|W_{k}(\zeta, \omega)\right| \leq W_{k}(\zeta, \zeta)=\theta_{k}$; that $W_{k}(U(\zeta), U(\omega))=W_{k}(\zeta, \omega)$ for any unitary transformation $U$; and that $W_{k}(\zeta, \cdot)$ is constant on each parallel of $\mathbb{S}$ orthogonal to $\zeta$ with respect to $\langle\cdot, \cdot\rangle$; all for any $\zeta, \omega \in \mathbb{S}$.

Extending to $\mathbb{B}$ by homogeneity as above and summing as in (11) using (4), we obtain

$$
\sum_{k=0}^{\infty} W_{k}(z, w)=\frac{1}{(1-\langle z, w\rangle)^{N}}=\mathcal{C}(z, w), \quad z, w \in \mathbb{B},
$$

which is the Cauchy-Szegő kernel for $\mathbb{B}$.

Going back to the zonal harmonics, it is well-known that the $Z_{m}(\xi, \eta)$ are multiples of the Gegenbauer (ultraspherical) polynomials $G_{n}^{\lambda}$, which are defined by the generating function

$$
\frac{1}{\left(1-2 v \tau+\tau^{2}\right)^{\kappa}}=\sum_{n=0}^{\infty} G_{n}^{\kappa}(v) \tau^{n}
$$

whose denominator is a power of the denominator of the Poisson kernel. Precisely,

$$
Z_{m}(\xi, \eta)=h_{m} \frac{(1)_{m}}{(n-2)_{m}} G_{n}^{n / 2-1}(\xi \cdot \eta)=\frac{n-2+2 m}{n-2} G_{n}^{n / 2-1}(\xi \cdot \eta)
$$

for $n>2$; see [11, p. 403]. This shows another similarity between the holomorphic and harmonic cases. The kernels $W_{k}$ and $Z_{m}$ depend on their variables via the associated inner product of the underlying space.

Remark 3.1. Yet there is an inherent difference between the harmonic and holomorphic cases due to the difference between the $\mathcal{H}_{m}$ and their holomorphic counterparts, and their dimensions $h_{m}$ and $\theta_{k}$.

The kernels $W_{k}$ and $Z_{m}$ remain reproducing kernels when their domains are extended from $\mathbb{S}$ to $\mathbb{B}$, because (5) is satisfied with the $r_{j} a_{j}$ replacing $a_{j}$, where $r_{j}=\left|\lambda_{j}\right|$ for $\lambda_{j} \in \mathbb{B}$. 


\section{Bergman spaces}

Definition 4.1. Let $q>-1$ and $p \geq 1$. The weighted Bergman space $A_{q}^{p}$ is the space of all $f \in H(\mathbb{B})$ that lie also in $L_{q}^{p}$. Similarly, the weighted harmonic Bergman space $b_{q}^{p}$ is the space of all $u \in h(\mathbb{B})$ that lie also in $L_{q}^{p}$. The norms of $A_{q}^{p}$ and $b_{q}^{p}$ are those of $L_{q}^{p}$, and the same is true of the inner products for $p=2$.

Polynomials and functions bounded on $\mathbb{B}$ belong to $L_{q}^{p}$ whenever $\nu_{q}$ is finite; this explains the condition $q>-1$. In Section 8, we have the variant (25) of this inequality for Besov spaces when $q \leq-1$.

The restriction to $p \geq 1$ is for having a unified presentation of both holomorphic and harmonic spaces. The reason is that $|f|^{p}$ is subharmonic for all $p>0$ when $f \in H(\mathbb{B})$, but only for $p \geq 1$ when $f \in h(\mathbb{B})$. The proof of [10, Thm. 1.1] shows where exactly this property is used.

Bergman spaces of either kind are Banach spaces, and when $p=2$, reproducing kernel Hilbert spaces. Using the orthonormal basis

$$
\left\{\sqrt{\frac{(1+N+q)_{|\alpha|}}{\alpha !}} z^{\alpha}: \alpha \in \mathbb{N}^{N}\right\}
$$

and Theorem 2.6, the reproducing kernel of $A_{q}^{2}$ for $q>-1$ is computed as

$$
\begin{aligned}
K_{q}(z, w) & =\frac{1}{(1-\langle z, w\rangle)^{1+N+q}}=\sum_{k=0}^{\infty} \frac{(1+N+q)_{k}}{(1)_{k}}\langle z, w\rangle^{k} \\
& =\sum_{k=0}^{\infty} \frac{(1+N+q)_{k}}{(N)_{k}} W_{k}(z, w)=\sum_{k=0}^{\infty} c_{k}(q) W_{k}(z, w),
\end{aligned}
$$

where $z, w \in \mathbb{B}$, and the $c_{k}(q)$ are defined. We call the $K_{q}$ introduced so far binomial kernels.

Because of the lack of a simple summable basis, the computation of the reproducing kernels of harmonic Bergman spaces has to follow a different path. The reproducing kernel of $b_{q}^{2}$ for $q>-1$ is computed to be

$$
R_{q}(z, w)=\sum_{m=0}^{\infty} \frac{\left(1+\frac{n}{2}+q\right)_{m}}{\left(\frac{n}{2}\right)_{m}} Z_{m}(x, y)=\sum_{m=0}^{\infty} \gamma_{m}(q) Z_{m}(x, y),
$$

where $x, y \in \mathbb{B}$ and the $\gamma_{m}(q)$ are defined; see [22, Prop. 3]. Additionally see $[9,(3.1)]$ or $[8,(1)]$ for integer or real $q>-1$; see also $[19$, p. 25], [20], and [5, Ch. 8] for $q=0$.

When interpreted as above, the forms of $K_{q}$ and $R_{q}$ turn out to be exactly the same in even dimensions with the identification $n=2 N$. It would be interesting to know if the $R_{q}$ could be expressed in a form that resembles the binomial form of the $K_{q}$, at least for even $n$. 
There are other similarities. We have $c_{0}(q)=1=\gamma_{0}(q)$ for all $q$. More importantly,

$$
c_{k}(q) \sim k^{1+q} \quad \text { as } k \rightarrow \infty \quad \text { and } \quad \gamma_{m}(q) \sim m^{1+q} \quad \text { as } m \rightarrow \infty .
$$

An important property we utilize most is that $K_{q}$ and $R_{q}$ are both weighted infinite sums zonal harmonics or their equivalents, which are reproducing kernels on their own. A careful look at (15) shows that the $c_{k}(q)$ and the $K_{q}$ make sense for $q>-(1+N)$ although Bergman spaces are defined only for $q>-1$. For all such extended $q$, (17) still holds and yields that

$$
\left|K_{q}(z, w)\right| \leq C \sum_{k=0}^{\infty} k^{N+q}(R r)^{k}, \quad z, w \in \mathbb{B}, q>-(1+N) .
$$

This shows that the series giving $K_{q}$ converges absolutely and uniformly on compact subsets of $\mathbb{B} \times \mathbb{B}$. Thus by Corollary 2.5, additionally for each $q$ with $-(1+N)<q \leq-1, K_{q}$ is the reproducing kernel for a unique Hilbert space of holomorphic functions on $\mathbb{B}$; let's call these spaces $\mathcal{D}_{q}$. It is easy to know what this space is when $q=-1$; since $c_{k}(-1)=1$ for all $k$, by $(14), \mathcal{D}_{-1}$ is the Hardy space $H^{2}(\mathbb{B})$.

Similarly, the $\gamma_{m}(q)$ and the $R_{q}$ also make sense in a larger interval of $q$, this time for $q>-(1+n / 2)$. For all such extended $q$, (17) still holds, and with the help of (9) and (6), yields that

$$
\begin{aligned}
\left|R_{q}(x, y)\right| & \leq C \sum_{m=0}^{\infty} m^{1+q}(R r)^{m} Z_{m}(\xi, \xi)=C \sum_{m=0}^{\infty} m^{1+q}(R r)^{m} h_{m} \\
& \leq C \sum_{m=0}^{\infty} m^{n-1+q}(R r)^{m}, \quad x, y \in \mathbb{B}, q>-\left(1+\frac{n}{2}\right) .
\end{aligned}
$$

As above, the convergence is absolute and uniform on compact subsets which allows us to conclude that $R_{q}$ is the reproducing kernel of a unique Hilbert space of harmonic functions on $\mathbb{B}$ for each $-(1+n / 2)<q \leq-1$ as well; let's call these spaces $\Delta_{q}$. The space for $q=-1$ is known again since $\gamma_{m}(-1)=1$ for all $m$; by $(11), \Delta_{-1}$ is the harmonic Hardy space $h^{2}(\mathbb{B})$; see $[5$, Ch. 6]. The closed form (12) of its kernel, the Poisson kernel, is a binomial-type expression. We do not know whether or not the remaining $R_{q}$ for $q>-(1+n / 2)$ have such closed-form or binomial-type expressions.

Remark 4.2. We see here a small difference between the holomorphic and harmonic cases. Due to the different growth rates of $\theta_{k}$ and $h_{m}$ for large $k$ and $m$, the growth rates of the coefficients of $(R r)^{k}$ and $(R r)^{m}$ are different.

Two main points of interest are to extend this construction to all real values of $q$, and to describe the non-Bergman Hilbert spaces in a way similar to Bergman spaces, that is, through integral norms. 


\section{Homogeneous expansions and radial derivatives}

An $f \in H(\mathbb{B})$ and a $u \in h(\mathbb{B})$ has a homogeneous expansion in the form

$$
f=\sum_{k=0}^{\infty} f_{k}, \quad u=\sum_{m=0}^{\infty} u_{m}
$$

where $f_{k}$ is a polynomial of the form (13) and $u_{m} \in \mathcal{H}_{m}$, which converge absolutely and uniformly on compact subsets of $\mathbb{B}$; see [23, Thm. 1.5.6] and [5, Cor. 5.23]. Their radial derivatives take the form

$$
\mathcal{R} f(z)=\sum_{k=1}^{\infty} k f_{k}(z), \quad \mathcal{R} u(x)=\sum_{m=1}^{\infty} m u_{m}(x)
$$

by (3), which also clearly converge absolutely and uniformly on compact subsets of $\mathbb{B}$. Thus $\mathcal{R} f \in H(\mathbb{B})$ and $\mathcal{R} u \in h(\mathbb{B})$ as well.

Remark 5.1. For $j=1,2, \ldots, \mathcal{R}^{j}$ has the index of summation raised to the $j$ th power; so this power determines the order of the radial derivative. By raising the index to a power $t \in \mathbb{R}, t>0$, one often considers radial derivatives of fractional order.

Further, $\mathcal{R}(\langle z, w\rangle)=\langle z, w\rangle$, where $\mathcal{R}$ acts on the holomorphic variable $z$. Then

$$
\mathcal{R} K_{q}(z, w)=(1+N+q)\langle z, w\rangle K_{1+q}(z, w), \quad q>-(1+N),
$$

and in particular,

$$
K_{0}=\mathcal{C}+\frac{1}{N} \mathcal{R C}
$$

A similar statement for $R_{q}$ is

$$
R_{q}(x, y)=\frac{2}{n(1)_{q}}\left(\frac{d}{d t}\right)^{1+q}\left[t^{n / 2+q} \mathcal{P}(t x, y)\right]_{t=1}, \quad q \in \mathbb{N}
$$

see [22, p. 29]. In particular,

$$
R_{0}=\mathcal{P}+\frac{1}{\frac{n}{2}} \mathcal{R} \mathcal{P},
$$

where differentiation is applied only on one of the variables $x, y$, and it does not matter which by symmetry; see [5, p. 157].

So the radial derivative allows us to go from a Bergman Hilbert space to another one whose lower index is greater by a positive integer. It would be nice if the same passage could be done if the difference between the indices is not an integer, and also if it is negative. Easy modifications would also eliminate the unnecessary multiplicative constants above. The particular relations for $K_{0}$ and $R_{0}$ also suggest that combinations that do not annihilate constants are more useful than pure radial derivatives.

All these are possible if we have radial derivatives of order any real number $t$ that are suited to the particular forms of the reproducing kernels. 


\section{Besov kernels}

We are now ready to present the extensions of the reproducing kernels $K_{q}$ and $R_{q}$ to all real $q$. The key idea is to keep unchanged the growth rates expressed in (17) of the coefficients of $W_{k}$ and $Z_{m}$ in the series expansions of $K_{q}$ and $R_{q}$. We replace $c_{k}(q)$ and $\gamma_{m}(q)$ by new ones for $q \leq-(1+N)$ or $q \leq-(1+n / 2)$ that still satisfy (17).

The holomorphic case has already been dealt with in [6, p. 13]. Here we modify it only slightly to have $c_{0}(q)=1$.

Definition 6.1. We set

$$
c_{k}(q)=\frac{(1)_{k}^{2}}{(1-(N+q))_{k}(N)_{k}}, \quad q \leq-(1+N) .
$$

The harmonic case is done only recently in [12, Def. 3.1]; it is fashioned after the holomorphic case.

Definition 6.2. We set

$$
\gamma_{m}(q)=\frac{(1)_{m}^{2}}{\left(1-\left(\frac{n}{2}+q\right)\right)_{m}\left(\frac{n}{2}\right)_{m}}, \quad q \leq-\left(1+\frac{n}{2}\right) .
$$

Now $c_{0}(q)=1=\gamma_{0}(q)$ for all $q \in \mathbb{R}$. More importantly, for all $q \in \mathbb{R}$ and $k, m \in \mathbb{N}, c_{k}(q)>0$ and $\gamma_{m}(q)>0$. Using (1) and considering (15) and (16) in conjunction with Definitions 6.1 and 6.2, we easily see that (17) now holds for all $q \in \mathbb{R}$. Then for every $q \in \mathbb{R}$,

$$
K_{q}(z, w)=\sum_{k=0}^{\infty} c_{k}(q) W_{k}(z, w) \quad \text { and } \quad R_{q}(x, y)=\sum_{m=0}^{\infty} \gamma_{m}(q) Z_{m}(x, y)
$$

converge uniformly and absolutely on compact subsets of $\mathbb{B} \times \mathbb{B}$, are reproducing kernels on $\mathbb{B}$, and define unique Hilbert spaces of holomorphic or harmonic functions on $\mathbb{B}$. Let's again call these Hilbert spaces $\mathcal{D}_{q}$ and $\Delta_{q}$. Also for uniformity, let's set $\mathcal{D}_{q}$ as the Bergman space $A_{q}^{2}$ for $q>-1$, and let's set $\Delta_{q}$ as the Bergman space $b_{q}^{2}$ for $q>-1$. Evidently, all the $R_{q}$ are real-valued and symmetric in their two variables, and all the $K_{q}$ are sesquilinear.

It is easy to see that

$$
K_{q}(z, w)={ }_{2} F_{1}(1,1 ; 1-(N+q) ;\langle z, w\rangle), \quad q \leq-(1+N) .
$$

It is also classical that the Gegenbauer polynomials, and hence the zonal harmonics, are finite hypergeometric series, but we do not know whether or not weighted infinite sums of them, the $R_{q}$ for $q \leq-(1+n / 2)$, have representations in terms of some special functions.

Now for all $q \in \mathbb{R}$, we can write

$$
K_{q}(z, w)=\sum_{k=0}^{\infty} c_{k}(q) \frac{(N)_{k}}{(1)_{k}} \sum_{|\alpha|=k} \frac{k !}{\alpha !} z^{\alpha} \bar{w}^{\alpha}=\sum_{\alpha} \frac{c_{k}(q)(N)_{k}}{\alpha !} z^{\alpha} \bar{w}^{\alpha},
$$


which shows that $\|1\|_{\mathcal{D}_{q}}=1$ and

$$
\left\|z^{\alpha}\right\|_{\mathcal{D}_{q}}{ }^{2}=\frac{\alpha !}{c_{k}(q)(N)_{k}}, \quad q \in \mathbb{R} .
$$

Consequently, an $f(z)=\sum_{\alpha} f_{\alpha} z^{\alpha} \in H(\mathbb{B})$ belongs to $\mathcal{D}_{q}, q \in \mathbb{R}$, if and only if

$$
\|f\|_{\mathcal{D}_{q}}{ }^{2}=\sum_{\alpha}\left|f_{\alpha}\right|^{2} \frac{\alpha !}{c_{|\alpha|}(q)(N)_{|\alpha|}}<\infty .
$$

For an equivalent formulation in $\Delta_{q}$, we consider the breaking up (10) of $Z_{m}(x, y)$ into smaller pieces. Then

$$
R_{q}(x, y)=\sum_{m=0}^{\infty} \gamma_{m}(q) \sum_{j=1}^{h_{m}} Y_{m j}(x) \bar{Y}_{m j}(y)
$$

which shows that

$$
\left\|Y_{m j}\right\|_{\Delta_{q}}{ }^{2}=\frac{1}{\gamma_{m}(q)}, \quad q \in \mathbb{R}, j=1, \ldots, h_{m},
$$

and $\|1\|_{\Delta_{q}}=1 / \gamma_{0}(q)=1$. Moreover, each term $u_{m}$ in the homogeneous expansion of a $u \in h(\mathbb{B})$ has an expansion in terms of the basis $\left\{Y_{m 1}, \ldots, Y_{m h_{m}}\right\}$; that is,

$$
u(x)=\sum_{m=0}^{\infty} R^{m} u_{m}(\xi)=\sum_{m=0}^{\infty} R^{m} \sum_{j=1}^{h_{m}} u_{m j} Y_{m k}(\xi)=\sum_{m=0}^{\infty} \sum_{j=1}^{h_{m}} u_{m j} Y_{m k}(x),
$$

where

$$
u_{m j}=\frac{1}{R^{m}} \int_{\mathbb{S}} u_{m}(x) \overline{Y_{m j}(\xi)} d \sigma(\xi)
$$

independently of any $0<R<1$.

Theorem 6.3. Let $q \in \mathbb{R}$. A function $u \in h(\mathbb{B})$ belongs to $\Delta_{q}$ if and only if

$$
\|u\|_{\Delta_{q}}{ }^{2}=\sum_{m=0}^{\infty} \sum_{j=1}^{h_{m}} \frac{\left|u_{m j}\right|^{2}}{\gamma_{m}(q)}<\infty
$$

This results appears in [12, Thm. 3.8].

Going back to the holomorphic case, the counterpart of the $Y_{m j}(x)$ are the functions

$$
V_{\alpha}(z)=\frac{\sqrt{(N)_{|\alpha|}}}{\sqrt{\alpha !}} R^{|\alpha|} \zeta^{\alpha}
$$

that are orthonormal with respect to the inner product of $L^{2}(\sigma)$ by Proposition 2.1. For each $k=|\alpha|$, there are $\theta_{k}$ such functions, and

$$
\left\|V_{\alpha}\right\|_{\mathcal{D}_{q}}{ }^{2}=\frac{1}{c_{|\alpha|}(q)}, \quad q \in \mathbb{R},|\alpha|=k .
$$


An $f \in H(\mathbb{B})$ can be written as $f(z)=\sum_{\alpha} \tilde{f}_{\alpha} V_{\alpha}(z)$, and it belongs to $\mathcal{D}_{q}, q \in \mathbb{R}$, if and only if

$$
\|f\|_{\mathcal{D}_{q}}{ }^{2}=\sum_{\alpha} \frac{\left|\tilde{f}_{\alpha}\right|^{2}}{c_{|\alpha|}(q)}<\infty .
$$

The equivalent norms (23) and (24) are useful for another purpose too. Using them, it is easy to see that if $q_{1}<q_{2}$, then $\mathcal{D}_{q_{1}} \subset \mathcal{D}_{q_{2}}$ and $\Delta_{q_{1}} \subset \Delta_{q_{2}}$. Further, examples are constructed in [2, Ex. 2.4] and [12, Ex. 3.10] that show that these inclusions are proper.

\section{Radial differential operators}

We now take one step further the fractional-order radial derivatives that we have already noted.

Definition 7.1. Let $s, t \in \mathbb{R}$ be unrestricted. We define positive numbers $d_{k}(s, t)$ and operators $D_{s}^{t}$ on $H(\mathbb{B})$ by

$$
D_{s}^{t} f=\sum_{k=0}^{\infty} d_{k}(s, t) f_{k}=\sum_{k=0}^{\infty} \frac{c_{k}(s+t)}{c_{k}(s)} f_{k},
$$

and other positive numbers $\delta_{m}(s, t)$ and operators $D_{s}^{t}$ on $h(\mathbb{B})$ by

$$
D_{s}^{t} u=\sum_{m=0}^{\infty} \delta_{m}(s, t) u_{m}=\sum_{m=0}^{\infty} \frac{\gamma_{m}(s+t)}{\gamma_{m}(s)} u_{m},
$$

where $f \in H(\mathbb{B})$ and $u \in h(\mathbb{B})$ are given by their homogeneous expansions (20), and $c_{k}$ and $\gamma_{m}$ are from Definitions 6.1 and 6.2 and equations (15) and (16).

Definition 7.1 is the essence of the connection between the Bergman-Besov kernels and the radial differential operators that help characterize the spaces they generate.

For any $s \in \mathbb{R}, D_{s}^{0}=I$, the identity. If a function is homogeneous of order $l$, then any $D_{s}^{t}$ applied on it is also homogeneous of degree $l$; in particular $D_{s}^{t}\left(\mathcal{H}_{m}\right)=\mathcal{H}_{m}$. Further, $d_{k}(s, t) \sim k^{t}$ as $k \rightarrow \infty$ and $\delta_{m}(s, t) \sim m^{t}$ as $m \rightarrow \infty$ by (1). Then by [3, Thm. 5], an appropriate $D_{s}^{t}$ maps $H(\mathbb{B})$ or $h(\mathbb{B})$ into itself continuously, and hence $D_{s}^{t} f$ is also holomorphic and $D_{s}^{t} u$ is also harmonic on $\mathbb{B}$.

We select $d_{k}(s, t)$ and $\delta_{m}(s, t)$ in order to have

$$
D_{q}^{t} K_{q}(z, w)=K_{q+t}(z, w) \quad \text { and } \quad D_{q}^{t} R_{q}(x, y)=R_{q+t}(x, y), \quad q, t \in \mathbb{R},
$$

where $D_{s}^{t}$ are applied only on the first variable just like $\mathcal{R}$. These immediately generalize $(21)$ and $(22)$ in two $(t>0$ or $t<0)$ directions. Remark 5.1 hints that many choices are possible for the $d_{k}$ and the $\delta_{k}$. All authors have their own particular coefficients, but for many formulas our choices seem to be the most 
natural and exact. Thus the reproducing kernels of Hilbert spaces determine the particular natural forms of the radial differential operators acting on them.

The last two paragraphs show that every $D_{s}^{t}$ is a radial differential operator of fractional order $t \in \mathbb{R}$. In particular, $D_{-N}^{1}=I+\mathcal{R}$ for $H(\mathbb{B})$ and $D_{-n / 2}^{1}=I+\mathcal{R}$ for $h(\mathbb{B})$. The $D_{s}^{t}$ are actually integral operators when $t<0$.

Moreover, $d_{k}(s, t) \neq 0$ for all $k$ including $k=0$, and $\delta_{m}(s, t) \neq 0$ for all $m$ including $m=0$. It is also clear that $D_{s+t}^{v} D_{s}^{t}=D_{s}^{v+t}$ for all choices of $s, t, v \in \mathbb{R}$. Then every $D_{s}^{t}$ is invertible on $H(\mathbb{B})$ or $h(\mathbb{B})$ with two-sided inverse $\left(D_{s}^{t}\right)^{-1}=D_{s+t}^{-t}$. The usual radial derivative $\mathcal{R}$ obviously is not invertible.

\section{Besov spaces}

For $s, t \in \mathbb{R}$, let's define further operators $I_{s}^{t}$ by

$$
\begin{aligned}
& I_{s}^{t} f(z)=\left(1-|z|^{2}\right)^{t} D_{s}^{t} f(z), \quad f \in H(\mathbb{B}), \\
& I_{s}^{t} u(x)=\left(1-|x|^{2}\right)^{t} D_{s}^{t} u(x), \quad u \in h(\mathbb{B}) .
\end{aligned}
$$

Thus $I_{s}^{0}=I$ as well for any $s \in \mathbb{R}$. It turns out that the $I_{s}^{t}$ are more useful than the $D_{s}^{t}$.

Definition 8.1. Let $q \in \mathbb{R}, 1 \leq p<\infty$, and pick $s, t$ such that

$$
q+p t>-1 \text {. }
$$

The Besov space $B_{q}^{p}$ is the space of all $f \in H(\mathbb{B})$ for which $I_{s}^{t} f$ lies in $L_{q}^{p}$. The harmonic Besov space $b_{q}^{p}$ is the space of all $u \in h(\mathbb{B})$ for which $I_{s}^{t} u$ lies in $L_{q}^{p}$. We also define $\|f\|_{B_{q}^{p}}=\left\|I_{s}^{t} f\right\|_{L_{q}^{p}}$ and $\|u\|_{b_{q}^{p}}=\left\|I_{s}^{t} u\right\|_{L_{q}^{p}}$, and similarly for the inner products for $p=2$.

That this definition is independent of $s, t$ as long as (25) is satisfied and that different $s, t$ satisfying (25) give equivalent norms have been shown for the holomorphic case in $[6$, Thm. 5.12 (i)], which is an excellent source on the spaces $B_{q}^{p}$; see also [17, Rem. 5.4] for another proof. For the harmonic case, it is in [19, Thm. 3]; see also [12, Prop. 3.6].

A norm of the harmonic Besov space $b_{q}^{p}$ written explicitly takes the form

$$
\|f\|_{b_{q}^{p}}=\left(C_{q} \int_{\mathbb{B}}\left|D_{s}^{t} u(x)\right|^{p}\left(1-|x|^{2}\right)^{q+p t} d \nu(x)\right)^{1 / p} .
$$

For $B_{q}^{p}$, we just change $u$ to $f$ and $x$ to $z$. This formula explains why (25) is essential and that it replaces the condition $q>-1$ of Bergman spaces. The invertibility of $D_{s}^{t}$ yields that only the zero function has norm 0 , and hence the Besov norms are genuine norms.

The condition (25) ensures that Besov spaces contain all polynomials of the right kind (holomorphic or harmonic). In fact, such polynomials are dense in every Besov space; this follows from the fact that they are dense in Bergman spaces 
and the fact that the $D_{s}^{t}$ acting on homogeneous polynomials of an order produce polynomials of the same order.

Note that if $q>-1,(25)$ is satisfied with $t=0$, and then the Besov space is nothing but the Bergman space with the same parameters. Here the use of the parameter $q$ for Besov spaces is non-standard; we follow [15] and [12]. But our $q$ for Besov spaces is selected in such a way that it extends the $q$ of the Bergman spaces to values $q \leq-1$ in the most natural manner.

The equivalence of norms extends farther on Besov Hilbert spaces.

Theorem 8.2. Let $q \in \mathbb{R}$ be unrestricted. The space $B_{q}^{2}$ and the the space $\mathcal{D}_{q}$ coincide with equivalent norms. The space $b_{q}^{2}$ and the the space $\Delta_{q}$ coincide with equivalent norms.

This result is in $[6$, p. 35$]$ for the holomorphic case; it is in $[12$, Thm. 3.7] for the harmonic case.

Remark 8.3. We have noted above that $B_{-1}^{2}$ is the Hardy space $H^{2}(\mathbb{B})$. However, this identification does not extend to other $p$. It is known that $B_{-1}^{p} \subset H^{p}(\mathbb{B})$ for $1 \leq p<2$ and $H^{p}(\mathbb{B}) \subset B_{-1}^{2}$ for $2<p<\infty$, where the inclusions are proper; see $[14$, p. 336].

When $q \leq-1$, a positive-order $D_{s}^{t}$ is required by (25). The question whether or not $B_{q}^{2}$ can be characterized by an integral using $f$ itself has been raised and answered negatively for $q=-N$ in [4, p. 180]. This has been extended negatively to all $q<-1$ in [7, Thm. 4.3]. The answer clearly is positive for $q=-1$ using the surface measure $\sigma$ on $\mathbb{S}$ since $B_{-1}^{2}=H^{2}$.

It is inherent in Definition 8.1 that every $I_{s}^{t}$ satisfying (25) imbeds $B_{q}^{p}$ or $b_{q}^{p}$ into the Lebesgue class $L_{q}^{p}$. Imbedding spaces via the $I_{s}^{t}$ or their cousins is just as important as imbedding them via the inclusion; see [21, Thm. 2] and [16, Thm. 1.3]. The boundedness of maps in the reverse direction is of considerable interest for many properties of Besov spaces can be derived from them.

Definition 8.4. For $s \in \mathbb{R}$, the integral operators

$$
P_{s} \varphi(z)=\int_{\mathbb{B}} K_{s}(z, w) \varphi(w) d \nu_{s}(w) \quad \text { and } \quad Q_{s} \varphi(x)=\int_{\mathbb{B}} R_{s}(x, y) \varphi(y) d \nu_{s}(y)
$$

acting on $\varphi$ in suitable Lebesgue classes are called (extended) Bergman projections.

Theorem 8.5. For $1 \leq p<\infty, P_{s}$ is a bounded operator from $L_{q}^{p}$ onto $B_{q}^{p}$ if and only if

$$
q+1<p(s+1) .
$$

Given an s satisfying (26), if t satisfies (25), then

$$
P_{s} I_{s}^{t} f=\frac{1}{C_{s+t}} f, \quad f \in B_{q}^{p},
$$


where $C_{s+t}$ is as in (2) with the identification $n / 2=N$.

Note that (26) and (25) together imply that $s+t>-1$.

Theorem 8.5 says that the imbeddings $I_{s}^{t}$ for which (25) holds are right inverses for the Bergman projections $P_{s}$ for which (26) holds. In this most general form for the holomorphic Besov spaces, this result is in [15, Thm. 1.2]. For the harmonic Besov spaces, such a general theorem will appear in [13]. For the boundedness of $Q_{s}$ on the harmonic Bergman spaces on the upper half space, see [18, Thm. 4.3]. It is surprising that the equivalent condition for the boundedness of Bergman projections is the same for holomorphic or harmonic Bergman-Besov spaces on the ball or on the upper half space; it is (26).

The maps $P_{s}$ and $Q_{s}$ are true projections on subspaces $B_{q}^{p}$ or $b_{q}^{p}$ of $L_{q}^{p}$ for $q>-1$ and $s=q$. For other values of $q$ and $s, I_{s}^{t} P_{s}$ is a true projection onto the subspace $I_{s}^{t}\left(B_{q}^{p}\right)$ of $L_{q}^{p}$, which is an isomorphic copy of $B_{q}^{p}$ inside $L_{q}^{p}$; see [17, Cor. 5.6].

The relations in (27), when written explicitly, are integral representations for functions in $B_{q}^{p}$ specific to the space. They are

$$
f(z)=\frac{(1+s+t)_{N}}{(1)_{N}} \int_{\mathbb{B}} D_{s}^{t} f(w) K_{s}(z, w)\left(1-|w|^{2}\right)^{s+t} d \nu(w), \quad f \in B_{q}^{p}, z \in \mathbb{B},
$$

where $1 \leq p<\infty$, and $s$ and $t$ satisfy (26) and (25). It is the existence of formulas of this kind that make the Bergman projections so useful.

\section{Special cases}

By definition, for $q>-1$, Besov spaces are actually Bergman spaces. We call the range $q \leq-1$ the proper Besov range. There are other values of $q$ and $p$ for which the Besov spaces have special names and properties, especially for $p=2$. We have already noted that $B_{-1}^{2}=H^{2}$ and $b_{-1}^{2}=h^{2}$, and for $N=1$, the reproducing kernel $K_{-1}$ takes the form

$$
K_{-1}(z, w)=\frac{1}{1-z \bar{w}}, \quad z, w \in \mathbb{D} .
$$

A kernel that has the same form for $N>1$ is

$$
K_{-N}(z, w)=\frac{1}{1-\langle z, w\rangle}, \quad z, w \in \mathbb{B} .
$$

The corresponding space $B_{-N}^{2}$ is called the Drury-Arveson space. It is the space that is considered the right generalization of the Hardy space $H^{2}(\mathbb{D})$ of the disc to higher dimensions from the point of view of operator theory and Nevanlinna-Pick interpolation; see [4]. Note also that

$$
K_{-(1+N)}(z, w)=\frac{1}{\langle z, w\rangle} \log \frac{1}{1-\langle z, w\rangle} ;
$$


thus $B_{-(1+N)}^{2}$ is the Dirichlet space; that's why we have initially named the family $B_{q}^{2}$ as $\mathcal{D}_{q}$.

In the plane where $N=1, n=2$, and $\mathbb{C}=\mathbb{R}^{2}$, there are closer relations between the holomorphic and the harmonic cases. Now $\theta_{k}=1$ and $h_{m}=2$ for all $k$ and $m \geq 1 ; z=x$ and $w=y$; also $\langle z, w\rangle=z \bar{w}$ and $Z_{m}(x, y)=x^{m} \bar{y}^{m}+\bar{x}^{m} y^{m}$. Further

$$
\begin{aligned}
c_{m}(q)=\gamma_{m}(q) & = \begin{cases}\frac{(2+q)_{m}}{m !}, & \text { if } q>-2 ; \\
\frac{m !}{(-q)_{m}}, & \text { if } q \leq-2 ;\end{cases} \\
K_{q}(z, w) & = \begin{cases}\frac{1}{(1-z \bar{w})^{2+q}}, & \text { if } q>-2 ; \\
{ }_{2} F_{1}(1,1 ;-q ; z \bar{w}), & \text { if } q \leq-2 ;\end{cases} \\
R_{q}(x, y) & =K_{q}(x, y)+\overline{K_{q}(x, y)}-1 .
\end{aligned}
$$

At some critical values of $q$, certain crucial changes occur at the reproducing kernels $K_{q}$ and $R_{q}$. There are 2 critical values of $q$ in the holomorphic category. At $q=-1$, we pass between Bergman and proper Besov spaces. At $q=-(1+N)$, we pass between binomial (Bergman-type) kernels and hypergeometric kernels. This value $q=-(1+N)$ is also the passage point between unbounded kernels and bounded kernels. By (18), the series giving $K_{q}$ converges absolutely and uniformly on $\overline{\mathbb{B}} \times \overline{\mathbb{B}}$ for $q<-(1+N)$, which yields that the spaces $B_{q}^{2}$ for such $q$ consist of bounded holomorphic functions on $\mathbb{B}$ that extend continuously to $\mathbb{S}$.

By contrast, in the harmonic category, there are 3 critical values of $q$. This difference arises from the dissimilarities expressed in Remarks 3.1 and 4.2. The behavior at $q=-1$ is as expected; we pass between Bergman and proper Besov spaces, that is, between spaces that require derivatives in their integral norms and those that do not. At $q=-(1+n / 2)$, we pass between Bergman-type kernels and kernels that are not. At $q=-n$, we pass between unbounded kernels and bounded ones. Now by (19), the series giving $R_{q}$ converges absolutely and uniformly on $\overline{\mathbb{B}} \times \overline{\mathbb{B}}$ for $q<-n$, which yields that the spaces $b_{q}^{2}$ for such $q$ consist of bounded harmonic functions on $\mathbb{B}$ that extend continuously to $\mathbb{S}$. When $n=2$, the latter two critical values are merged and is the same as the critical value for the holomorphic category with $N=1$.

Acknowledgement. The author thanks A. E. Üreyen of Anadolu University and S. Gergün of Çankaya University for their input on harmonic spaces.

\section{References}

1. J. Agler and J. E. McCarthy, Pick Interpolation and Hilbert Function Spaces, Grad. Stud. Math., vol. 44, Amer. Math. Soc., Providence, 2002. 
2. D. Alpay and H. T. Kaptanoğlu, Gleason's problem and homogeneous interpolation in Hardy and Dirichlet-type spaces, J. Math. Anal. Appl. 276 (2002), 654-672.

3. J. Arazy, Boundedness and compactness of generalized Hankel operators on bounded symmetric domains, J. Funct. Anal. 137 (1996), 97-151.

4. W. Arveson, Subalgebras of $C^{*}$-algebras III: multivariable operator theory, Acta Math. 181 (1998), 159-228.

5. S. Axler, P. Bourdon, and W. Ramey, Harmonic Function Theory, Grad. Texts in Math., vol. 137, Springer, New York, 1992.

6. F. Beatrous and J. Burbea, Holomorphic Sobolev Spaces on the Ball, Dissertationes Math. Soc. 276 (1989), 57 pp.

7. __ On multipliers for Hardy-Sobolev spaces, Proc. Amer. Math. Soc. 136 (2008), 2125-2133.

8. O. Blasco and S. Pérez-Esteva, $L^{p}$ continuity of projectors of weighted harmonic Bergman spaces, Collect. Math. 51 (2000), 49-58.

9. R. R. Coifman and R. Rochberg, Representation theorems for holomorphic and harmonic functions in $L^{p}$, Astérisque 77 (1980), 12-66.

10. P. Duren and A. Schuster, Bergman Spaces, Math. Surveys Monogr., vol. 100, Amer. Math. Soc., Providence, 2004.

11. G. B. Folland, Spherical harmonic expansion of the Poisson-Szegö kernel for the ball, Proc. Amer. Math. Soc. 47 (1975), 401-408.

12. S. Gergün, H. T. Kaptanoğlu, and A. E Üreyen, Reproducing kernels for harmonic Besov spaces on the ball, C. R. Math. Acad. Sci. Paris 347 (2009), 735-738.

13. Harmonic Besov spaces on the ball, to appear.

14. D. Girela and J. Á. Peláez, Carleson measures, multipliers and integration operators for spaces of Dirichlet type, J. Funct. Anal. 241 (2006), 334-358.

15. H. T. Kaptanoğlu, Bergman projections on Besov spaces on balls, Illinois J. Math. 49 (2005), 385-403.

16. Carleson measures for Besov spaces on the ball with applications, J. Funct. Anal. 250 (2007), 483-520.

17. H. T. Kaptanoğlu and A. E Üreyen, Analytic properties of Besov spaces via Bergman projections, Contemp. Math. 455 (2008), 169-182.

18. H. Koo, K. Nam and H. Yi, Weighted harmonic Bergman functions on half-spaces, $J$. Korean Math. Soc. 42 (2005), 975-1002.

19. E. Ligocka, On the reproducing kernel for harmonic functions and the space of Bloch harmonic functions on the unit ball in $\mathbb{R}^{n}$, Studia Math. 87 (1987), 23-32.

20. E. Ligocka, Corrigendum to the paper "On the reproducing kernel for harmonic functions and the space of Bloch harmonic functions on the unit ball in $\mathbb{R}^{n}$ ", Studia Math. 101 (1992), 319.

21. D. H. Luecking, Embedding theorems for spaces of analytic functions via Khinchine's inequality, Michigan Math. J. 40 (1993), 333-358.

22. J. Miao, Reproducing kernels for harmonic Bergman spaces of the unit ball, Monatsh. Math. 125 (1998), 25-35.

23. W. Rudin, Function Theory in the Unit Ball of $\mathbb{C}^{n}$, Grundlehren Math. Wiss., vol. 241, Springer, New York, 1980.

24. F. G. Tricomi and Erdélyi, The asymptotic expansion of a ratio of Gamma functions, Pacific J. Math. 1 (1951), 133-142. 
H. Turgay Kaptanoğlu

E-MAIL: kaptan@fen.bilkent.edu.tr ADDRESS: Bilkent University, Department of Mathematics, 06800 Ankara, Turkey. URL: http://www.fen.bilkent.edu.tr/ kaptan/ 